\title{
On $\mathscr{C}$-coherent rings, strongly $\mathscr{C}$-coherent rings and $\mathscr{C}$-semihereditary rings
}

\author{
Zhu Zhanmin (10 \\ Department of Mathematics, Jiaxing University, Jiaxing, Zhejiang Province, 314001, P.R.China
}

\begin{abstract}
Let $R$ be a ring and $\mathscr{C}$ be a class of some finitely presented left $R$-modules. A left $R$ module $M$ is called $\mathscr{C}$-injective if $\operatorname{Ext}_{R}^{1}(C, M)=0$ for every $C \in \mathscr{C}$; a left $R$-module $M$ is called $\mathscr{C}$-projective if $\operatorname{Ext}_{R}^{1}(M, E)=0$ for any $\mathscr{C}$-injective module $E$. $R$ is called left $\mathscr{C}$-coherent if every $C \in \mathscr{C}$ is 2-presented; $R$ is called left strongly $\mathscr{C}$-coherent, if whenever $0 \rightarrow K \rightarrow P \rightarrow C \rightarrow 0$ is exact, where $C \in \mathscr{C}$ and $P$ is finitely generated projective, then $K$ is $\mathscr{C}$-projective; a ring $R$ is called left $\mathscr{C}$-semihereditary, if whenever $0 \rightarrow K \rightarrow P \rightarrow C \rightarrow 0$ is exact, where $C \in \mathscr{C}, P$ is finitely generated projective, then $K$ is projective. In this paper, we give some new characterizations and properties of left $\mathscr{C}$-coherent rings, left strongly $\mathscr{C}$-coherent rings and left $\mathscr{C}$-semihereditary rings.
\end{abstract}

Mathematics Subject Classification (2010). 16D40, 16D50, 16E60, 16 P70.

Keywords. $\mathscr{C}$-coherent ring, strongly $\mathscr{C}$-coherent ring, $\mathscr{C}$-semihereditary ring.

\section{Introduction}

Recall that a ring $R$ is said to be left coherent $[1,19]$ if every finitely generated left ideal of $R$ is finitely presented, a ring $R$ is said to be left semihereditary if every finitely generated left ideal of $R$ is projective. Coherent rings, semihereditary rings and their generalizations have been studied extensively by many authors (see, for example, $[1,2$, $4,6,11,13-15,19,24,26])$. In [27], we introduced the concepts of left $\mathscr{C}$-coherent rings and left $\mathscr{C}$-semihereditary rings, and in [28], we introduced the concept of left strongly $\mathscr{C}$-coherent rings. Let $\mathscr{C}$ be a class of some finitely presented left $R$-modules. Following [27], a ring $R$ is called left $\mathscr{C}$-coherent if every $C \in \mathscr{C}$ is 2-presented; a ring $R$ is called left $\mathscr{C}$-semihereditary, if whenever $0 \rightarrow K \rightarrow P \rightarrow C \rightarrow 0$ is exact, where $C \in \mathscr{C}, P$ is finitely generated projective, then $K$ is projective. To characterize left $\mathscr{C}$-coherent rings and left $\mathscr{C}$-semihereditary rings, in [27], we also introduced the concepts of $\mathscr{C}$-injective modules and $\mathscr{C}$-flat modules. According to [27], a left $R$-module $M$ is called $\mathscr{C}$-injective if $\operatorname{Ext}_{R}^{1}(C, M)=0$ for every $C \in \mathscr{C}$, a right $R$-module $M$ is called $\mathscr{C}$-flat if $\operatorname{Tor}_{1}^{R}(M, C)=0$ for every $C \in \mathscr{C}$. In [28], we introduced the concepts of $\mathscr{C}$-projective modules and left strongly $\mathscr{C}$-coherent rings. Following [28], a left $R$-module $M$ is called $\mathscr{C}$-projective if $\operatorname{Ext}_{R}^{1}(M, E)=0$ for any $\mathscr{C}$-injective module $E$; a ring $R$ is called left strongly $\mathscr{C}$-coherent, if whenever $0 \rightarrow K \rightarrow P \rightarrow C \rightarrow 0$ is exact, where $C \in \mathscr{C}$ and $P$ is finitely generated

Email address: zhuzhanminzjxu@hotmail.com

Received: 30.05.2018; Accepted: 05.04.2019 
projective, then $K$ is $\mathscr{C}$-projective. We shall denote the class of $\mathscr{C}$-flat (resp., $\mathscr{C}$-injective, $\mathscr{C}$-projective) modules by $\mathscr{C} \mathcal{F}$ (resp., $\mathscr{C} J, \mathscr{C} \mathcal{P}$ ).

In this article, we continues to study left $\mathscr{C}$-coherent rings, left strongly $\mathscr{C}$-coherent rings and left $\mathscr{C}$-semihereditary rings. Series characterizations and properties of these rings will be given respectively.

Next, we recall some known notions and facts needed in the sequel.

Given a class $\mathscr{L}$ of $R$-modules, we shall denote by $\mathscr{L}^{\perp}=\left\{M: \operatorname{Ext}_{R}^{1}(L, M)=0, L \in \mathscr{L}\right\}$ the right orthogonal class of $\mathscr{L}$, and by ${ }^{\perp} \mathscr{L}=\left\{M: \operatorname{Ext}_{R}^{1}(M, L)=0, L \in \mathscr{L}\right\}$ the left orthogonal class of $\mathscr{L}$.

Let $\mathcal{F}$ be a class of $R$-modules and $M$ an $R$-module. Following [9], we say that a homomorphism $\varphi: M \rightarrow F$ where $F \in \mathcal{F}$ is an $\mathcal{F}$-preenvelope of $M$ if for any morphism $f: M \rightarrow F^{\prime}$ with $F^{\prime} \in \mathcal{F}$, there is a $g: F \rightarrow F^{\prime}$ such that $g \varphi=f$. An $\mathcal{F}$-preenvelope $\varphi: M \rightarrow F$ is said to be an $\mathcal{F}$-envelope if every endomorphism $g: F \rightarrow F$ such that $g \varphi=\varphi$ is an isomorphism. Dually, we have the definitions of $\mathcal{F}$-precovers and $\mathcal{F}$-covers. $\mathcal{F}$-envelopes (F-covers) may not exist in general, but if they exist, they are unique up to isomorphism. It is easy to see that every $\mathscr{C}$-injective preenvelope is monic, and every $\mathscr{C}$-projective precover is epic.

Following [9], a pair $(\mathscr{A}, \mathscr{B})$ of classes of $R$-modules is called a cotorsion pair if $\mathscr{A}^{\perp}=\mathscr{B}$ and $\perp \mathscr{B}=\mathscr{A}$. A cotorsion pair $(\mathscr{A}, \mathscr{B})$ is called hereditary [10, Definition 1.1] if whenever $0 \rightarrow A^{\prime} \rightarrow A \rightarrow A^{\prime \prime} \rightarrow 0$ is exact with $A, A^{\prime \prime} \in \mathscr{A}$ then $A^{\prime}$ is also in $\mathscr{A}$. By [10, Proposition 1.2], a cotorsion pair $(\mathscr{A}, \mathscr{B})$ is hereditary if and only if whenever $0 \rightarrow B^{\prime} \rightarrow B \rightarrow B^{\prime \prime} \rightarrow 0$ is exact with $B^{\prime}, B \in \mathscr{B}$ then $B^{\prime \prime}$ is also in $\mathscr{B}$. A cotorsion pair $(\mathscr{A}, \mathscr{B})$ is called perfect [10] if every $R$-module has an $\mathscr{A}$-cover and a $\mathscr{B}$-envelope. A cotorsion pair $(\mathscr{A}, \mathscr{B})$ is called complete (see [9, Definition 7.16] and [20, Lemma 1.13]) if for any $R$-module $M$, there are exact sequences $0 \rightarrow M \rightarrow B \rightarrow A \rightarrow 0$ with $A \in \mathscr{A}$ and $B \in \mathscr{B}$, and $0 \rightarrow B^{\prime} \rightarrow A^{\prime} \rightarrow M \rightarrow 0$ with $A^{\prime} \in \mathscr{A}$ and $B^{\prime} \in \mathscr{B}$.

Throughout this paper, $R$ is an associative ring with identity and all modules considered are unitary, $\mathscr{C}$ is a class of some finitely presented left $R$-modules. For any $R$-module $M$, $E(M)$ will denote the injective envelope of $M, M^{+}=\operatorname{Hom}(M, \mathbb{Q} / \mathbb{Z})$ will be the character module of $M$ and $M^{*}=\operatorname{Hom}(M, R)$ will be the dual module of $M$.

\section{2. $\mathscr{C}$-coherent rings}

Theorem 2.1. The following statements are equivalent for a ring $R$ :

(1) $R$ is a left $\mathscr{C}$-coherent ring.

(2) For any projective left $R$-module $P, P^{*}$ is $\mathscr{C}$-flat.

(3) For any free left $R$-module $F, F^{*}$ is $\mathscr{C}$-flat.

Proof. $(1) \Rightarrow(2)$. For any projective left $R$-module $P$, there is an index set $I$ and an $R$-module $Q$ such that $P \oplus Q \cong R^{(I)}$. So we have $P^{*} \oplus Q^{*} \cong\left(R^{(I)}\right)^{*} \cong R^{I}$, and thus $P^{*}$ is $\mathscr{C}$-flat by [27, Theorem 3.3(4) and Proposition 2.6].

$(2) \Rightarrow(3)$. It is clear.

$(3) \Rightarrow(1)$. Let $I$ be any index set. Then by $(3), R^{I} \cong\left(R^{(I)}\right)^{*}$ is $\mathscr{C}$-flat, and so $R$ is $\mathscr{C}$-coherent by [27, Theorem 3.3(4)].

Recall that a left $R$-module $M$ is said to be FP-injective [19] if $\operatorname{Ext}_{R}^{1}(A, M)=0$ for every finitely presented left $R$-module $A$; a left $R$-module $M$ is said to be P-injective [16] if every homomorphism from a principal left ideal of $R$ to $M$ can be extended to a homomorphism of $R$ to $M$, it is easy to see that a left $R$-module $M$ is P-injective if and only if $\operatorname{Ext}_{R}^{1}(R / R a, M)=0$ for any $a \in R$. We recall also that a left $R$-module $M$ is said to be FI-injective [13] (resp., D-injective [14], copure injective [8] ) if $\operatorname{Ext}_{R}^{1}(G, M)=0$ for every FP-injective (resp., P-injective, injective) left $R$-module $G$; a right $R$-module $N$ is said to be FI-flat [13] (resp., D-flat [14], copure flat [8]) if $\operatorname{Tor}_{1}^{R}(N, G)=0$ for every 
FP-injective (resp., P-injective, injective) left $R$-module $G$. Inspired by these concepts, we have the following concepts.

Definition 2.2. A left $R$-module $M$ is said to be $\mathscr{C}$ I-injective if $\operatorname{Ext}_{R}^{1}(G, M)=0$ for every $\mathscr{C}$-injective left $R$-module $G$; a right $R$-module $F$ is said to be $\mathscr{C}$ I-flat if $\operatorname{Tor}_{1}^{R}(F, G)=0$ for every $\mathscr{C}$-injective left $R$-module $G$.

Proposition 2.3. The following statements are equivalent for a left $R$-module $M$ :

(1) $M$ is $\mathscr{C}$ I-injective.

(2) For every exact sequence $0 \rightarrow M \rightarrow E \rightarrow L \rightarrow 0$ with $E \mathscr{C}$-injective, $E \rightarrow L$ is a $\mathscr{C}$-injective precover of $L$.

(3) $M$ is the kernel of a $\mathscr{C}$-injective precover $f: E \rightarrow L$ with $E$ injective.

(4) $M$ is injective with respect to every exact sequence $0 \rightarrow A \rightarrow B \rightarrow C \rightarrow 0$ with $C$ $\mathscr{C}$-injective.

Proof. $(1) \Rightarrow(2)$ and $(1) \Rightarrow(4)$ are clear.

$(2) \Rightarrow(3)$. It follows from the exact sequence $0 \rightarrow M \rightarrow E(M) \rightarrow E(M) / M \rightarrow 0$.

$(3) \Rightarrow(1)$. Let $M$ be the kernel of a $\mathscr{C}$-injective precover $f: E \rightarrow L$ with $E$ injective. Then $f: E \rightarrow \operatorname{im}(f)$ is a $\mathscr{C}$-injective precover, so, for any $\mathscr{C}$-injective module $N$, the map $\operatorname{Hom}(N, E) \rightarrow \operatorname{Hom}(N, \operatorname{im}(f))$ is epic and hence the map $\operatorname{Hom}(N, E) \rightarrow \operatorname{Hom}(N, E / M)$ is epic. Thus, by the exactness of the sequence $0 \rightarrow \operatorname{Hom}(N, E) \rightarrow \operatorname{Hom}(N, E / M) \rightarrow$ $\operatorname{Ext}_{R}^{1}(N, M) \rightarrow 0$, we have $\operatorname{Ext}_{R}^{1}(N, M)=0$.

$(4) \Rightarrow(1)$. For any $\mathscr{C}$-injective module $N$, there exists an exact sequence $0 \rightarrow K \rightarrow$ $P \rightarrow N \rightarrow 0$, where $P$ is projective. Hence we get an exact sequence $\operatorname{Hom}(P, M) \rightarrow$ $\operatorname{Hom}(K, M) \rightarrow \operatorname{Ext}_{R}^{1}(N, M) \rightarrow \operatorname{Ext}_{R}^{1}(P, M)=0$, and thus $\operatorname{Ext}_{R}^{1}(N, M)=0$ by (4). Therefore, $M$ is $\mathscr{C}$ I-injective.

Remark 2.4. Since the class of all $\mathscr{C}$-injective modules is closed under extensions, by Wakamutsu's Lemma (see [23, Lemma 2.1.1]), any kernel of a $\mathscr{C}$-injective cover is $\mathscr{C} \mathrm{I}$ injective .

Recall that a left $R$-module $M$ is called reduced [9] if $M$ has no nonzero injective submodules.

Proposition 2.5. Let $R$ be a left $\mathscr{C}$-coherent ring. Then the following statements are equivalent for a left $R$-module $M$ :

(1) $M$ is a reduced $\mathscr{C}$ I-injective module.

(2) $M$ is the kernel of a $\mathscr{C}$-injective cover $f: E \rightarrow L$ with $E$ injective.

Proof. $(1) \Rightarrow(2)$. Since $M$ is $\mathscr{C}$ I-injective, by proposition 2.3 , the natural mapping $\pi$ : $E(M) \rightarrow E(M) / M$ is a $\mathscr{C}$-injective precover. Since $R$ is left $\mathscr{C}$-coherent, by [27, Corollary 3.7], $E(M) / M$ has a $\mathscr{C}$-injective cover. Note that there is no nonzero summand $K$ of $E(M)$ contained in $M$ as $M$ is reduced, by [23, Corollary 1.2.8], $\pi: E(M) \rightarrow E(M) / M$ is a $\mathscr{C}$-injective cover.

$(2) \Rightarrow(1)$. Let $M$ be the kernel of a $\mathscr{C}$-injective cover $f: E \rightarrow L$ with $E$ injective. Then by proposition $2.3(3), M$ is a $\mathscr{C}$ I-injective module. Now let $K$ be an injective submodule of $M$. Suppose $E=K \oplus N, p: E \rightarrow N$ is the projective and $i: N \rightarrow E$ is the inclusion for some submodule $N$ of $M$. It is easy to see that $f(i p)=f$ since $f(K)=0$. So $i p$ is an isomorphism since $f$ is a cover. Thus $i$ is epic and hence $E=N, K=0$. Therefore $M$ is reduced.

Recall that a submodule $A$ of left $R$-module $B$ is said to be a pure submodule if for all right $R$-module $M$, the induced map $M \otimes_{R} A \rightarrow M \otimes_{R} B$ is monic, or equivalently, every finitely presented left $R$-module is projective with respect to the exact sequence $0 \rightarrow A \rightarrow B \rightarrow B / A \rightarrow 0$. In this case, the exact sequence $0 \rightarrow A \rightarrow B \rightarrow B / A \rightarrow 0$ 
is called pure exact. An exact sequence $0 \rightarrow A \rightarrow B \rightarrow L \rightarrow 0$ is called $R D$-exact [14] if, for any $a \in R, R / R a$ is projective with respect to this sequence. We call a short exact sequence of left $R$-modules $0 \rightarrow A \rightarrow B \rightarrow L \rightarrow 0 \mathscr{C}$-pure exact if every $C \in \mathscr{C}$ is projective with respect to this sequence. Let $A$ be a submodule of $B$, if the short exact sequence of left $R$-modules $0 \rightarrow A \rightarrow B \rightarrow B / A \rightarrow 0$ is $\mathscr{C}$-pure exact, then we call $A$ a $\mathscr{C}$-pure submodule of $B$ and $B / A$ a $\mathscr{C}$-pure quotient module of $B$.

Next, we give some characterizations of $\mathscr{C}$-injective modules.

Theorem 2.6. Let $M$ be a left $R$-module, then the following statements are equivalent:

(1) $M$ is $\mathscr{C}$-injective.

(2) $M$ is injective with respect to every exact sequence $0 \rightarrow A \rightarrow B \rightarrow C \rightarrow 0$ of left $R$-modules with $C \in \mathscr{C}$.

(3) $M$ is injective with respect to every exact sequence $0 \rightarrow K \rightarrow P \rightarrow C \rightarrow 0$ of left $R$-modules with $C \in \mathscr{C}$ and $P$ finitely generated projective.

(4) Every exact sequence $0 \rightarrow M \rightarrow M^{\prime} \rightarrow M^{\prime \prime} \rightarrow 0$ is $\mathscr{C}$-pure.

(5) There exists a $\mathscr{C}$-pure exact sequence $0 \rightarrow M \rightarrow M^{\prime} \rightarrow M^{\prime \prime} \rightarrow 0$ of left $R$-modules with $M^{\prime}$ injective.

(6) There exists a $\mathscr{C}$-pure exact sequence $0 \rightarrow M \rightarrow M^{\prime} \rightarrow M^{\prime \prime} \rightarrow 0$ of left $R$-modules with $M^{\prime}$ FP-injective.

(7) There exists a $\mathscr{C}$-pure exact sequence $0 \rightarrow M \rightarrow M^{\prime} \rightarrow M^{\prime \prime} \rightarrow 0$ of left $R$-modules with $M^{\prime} \mathscr{C}$-injective.

Proof. (1) $\Rightarrow(2)$. It follows from the exact sequence

$$
\operatorname{Hom}(B, M) \rightarrow \operatorname{Hom}(A, M) \rightarrow \operatorname{Ext}_{R}^{1}(C, M)=0 .
$$

$(2) \Rightarrow(3)$. It is obvious.

$(3) \Rightarrow(1)$. It follows from the exact sequence

$$
\operatorname{Hom}(P, M) \rightarrow \operatorname{Hom}(K, M) \rightarrow \operatorname{Ext}_{R}^{1}(C, M) \rightarrow \operatorname{Ext}_{R}^{1}(P, M)=0 .
$$

$(1) \Rightarrow(4)$. Assume (1). Then we have an exact sequence $\operatorname{Hom}\left(C, M^{\prime}\right) \rightarrow \operatorname{Hom}\left(C, M^{\prime \prime}\right) \rightarrow$ $\operatorname{Ext}_{R}^{1}(C, M)=0$ for every $C \in \mathscr{C}$, and so (4) follows.

$(4) \Rightarrow(5) \Rightarrow(6) \Rightarrow(7)$ is obvious.

$(7) \Rightarrow(1)$. By $(7)$, we have a $\mathscr{C}$-pure exact sequence $0 \rightarrow M \rightarrow M^{\prime} \stackrel{f}{\rightarrow} M^{\prime \prime} \rightarrow 0$ of left $R$-modules where $M^{\prime}$ is $\mathscr{C}$-injective, and so, for each $C \in \mathscr{C}$, we have an exact sequence $\operatorname{Hom}\left(C, M^{\prime}\right) \stackrel{f_{*}}{\rightarrow} \operatorname{Hom}\left(C, M^{\prime \prime}\right) \rightarrow \operatorname{Ext}_{R}^{1}(C, M) \rightarrow \operatorname{Ext}_{R}^{1}\left(C, M^{\prime}\right)=0$ with $f_{*}$ epic. Which implies that $\operatorname{Ext}_{R}^{1}(C, M)=0$, and (1) follows.

Recall that a left $R$-module $M$ is called pure injective [9, Definition 5.3.6] if it is injective with respect to every pure exact sequence of left $R$-modules; a left $R$-module $M$ is called $R D$-injective [14] if it is injective with respect to every RD-exact sequence of left $R$ modules. We call a left $R$-module $M \mathscr{C}$-pure injective if it is injective with respect to every $\mathscr{C}$-pure exact sequence of left $R$-modules.

Proposition 2.7. Let $R$ be a left $\mathscr{C}$-coherent ring. Then every $\mathscr{C}$-pure injective module $M$ has a $\mathscr{C}$-injective cover $f: N \rightarrow M$ with $N$ injective. Moreover, $\operatorname{Ker}(f)$ is a reduced $\mathscr{C} I$-injective left $R$-module.

Proof. By [27, Corollary 3.7], $M$ has a $\mathscr{C}$-injective cover $f: N \rightarrow M$. Since $N$ is $\mathscr{C}$ injective, by Theorem 2.6(4), the exact sequence $0 \rightarrow N \stackrel{i}{\rightarrow} E(N) \rightarrow E(N) / N \rightarrow 0$ is $\mathscr{C}$-pure exact, and so there exists $g: E(N) \rightarrow M$ such that $g i=f$. Note that $f$ is a cover, there exists $h: E(N) \rightarrow N$ such that $f h=g$. Thus $f h i=f$ and hence $h i$ is an isormorphism. It follows that $N$ is isomorphic to a direct summand of $E(N)$ and so $N$ is injective. By Proposition 2.5, $\operatorname{Ker}(f)$ is a reduced $\mathscr{C}$ I-injective left $R$-module. 
Theorem 2.8. Let $R$ be a left $\mathscr{C}$-coherent ring. Then a left $R$-module $M$ is $\mathscr{C}$ I-injective if and only if $M$ is a direct sum of an injective left $R$-module and a reduced $\mathscr{C}$ I-injective left $R$-module.

Proof. " $\Leftarrow$ ". It is clear.

" $\Rightarrow$ ". Let $M$ be a $\mathscr{C}$ I-injective left $R$-module . Then by Proposition 2.3, $E(M) \rightarrow$ $E(M) / M$ is a $\mathscr{C}$-injective precover. Since $R$ is left $\mathscr{C}$-coherent, $E(M) / M$ has a $\mathscr{C}$-injective cover $L \stackrel{g}{\rightarrow} E(M) / M$ by [27, Corollary 3.7], so we have the following commutative diagram with exact rows:

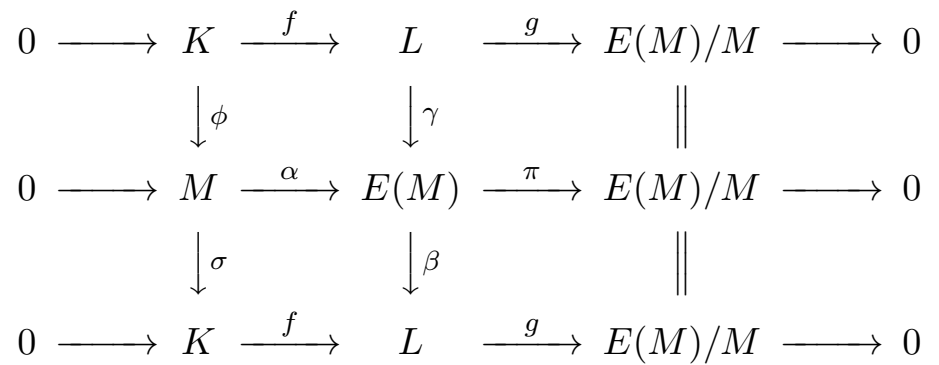

where $K$ is a reduced $\mathscr{C}$ I-injective left $R$-module by Proposition 2.5. Note that $g=g(\beta \gamma)$, we have that $\beta \gamma$ is an isomorphism, so $E(M)=\operatorname{Ker}(\beta) \oplus i m(\gamma)$, and thus $\operatorname{Ker}(\beta)$ is injective. Since $\sigma \phi$ is an isomorphism by the Five Lemma, we have that $M=\operatorname{Ker}(\sigma) \oplus$ $i m(\phi)$ and $i m(\phi) \cong K$. Moreover, by the Snake Lemma [17, Theorem 6.5], we have that $\operatorname{Ker}(\sigma) \cong \operatorname{Ker}(\beta)$ is injective. This completes the proof.

Proposition 2.9. Let $M$ be a right $R$-module. Then $M$ is $\mathscr{C}$ I-flat if and only if $M^{+}$is $\mathscr{C}$ I-injective.

Proof. It follows from the isomorphism $\operatorname{Tor}_{1}^{R}(M, G)^{+} \cong \operatorname{Ext}_{R}^{1}\left(G, M^{+}\right)$.

Corollary 2.10. A pure submodule of a $\mathscr{C} I$-flat module is $\mathscr{C} I$-flat.

Proof. Let $M$ be a $\mathscr{C}$ I-flat module and $M_{1}$ a pure submodule of $M$, then the pure exact sequence $0 \rightarrow M_{1} \rightarrow M \rightarrow M / M_{1} \rightarrow 0$ induces a split exact sequence $0 \rightarrow\left(M / M_{1}\right)^{+} \rightarrow$ $M^{+} \rightarrow M_{1}^{+} \rightarrow 0$. By Proposition $2.9, M^{+}$is $\mathscr{C}$ I-injective, so $M_{1}^{+}$is $\mathscr{C}$ I-injective, and hence $M_{1}$ is $\mathscr{C} \mathrm{I}$-flat by Proposition 2.9 again.

Proposition 2.11. Let $R$ be a ring and $\mathscr{C}$ be a class of some finitely presented left $R$ modules.

(1) If $M$ is a finitely presented $\mathscr{C}$ I-flat module, then it is a cokernet of a $\mathscr{C}$-flat preenvelope.

(2) If $R$ is left $\mathscr{C}$-coherent and $L$ is the cokernet of a $\mathscr{C}$ I-flat preenvelope $f: M \rightarrow F$, then $L$ is $\mathscr{C} I$-flat.

Proof. (1). Let $M$ be a finitely presented $\mathscr{C}$ I-flat module. Then there exists an exact sequence of right $R$-modules $0 \rightarrow K \rightarrow P \rightarrow M \rightarrow 0$ with $P$ finitely generated projective and $K$ finitely generated. We claim that $K \rightarrow P$ is a $\mathscr{C}$-flat preenvelope. In fact, for any $\mathscr{C}$-flat module $F$, we have $F^{+}$is $\mathscr{C}$-injective by [27, Theorem 2.7], and so $\operatorname{Tor}_{1}^{R}\left(M, F^{+}\right)=0$ since $M$ is $\mathscr{C}$ I-flat. Hence, we have the following commutative diagram with $\alpha$ monic:

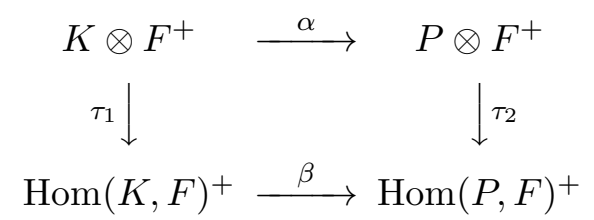

Since $K$ is finitely generated and $P$ is finitely presented, by [3, Lemma 2], $\tau_{1}$ is epic and $\tau_{2}$ is an isomorphism, this follows that $\beta$ is monic, and hence $\operatorname{Hom}(P, F) \rightarrow \operatorname{Hom}(K, F)$ is epic, as required. 
(2). There is an exact sequence $0 \rightarrow \operatorname{im}(f) \stackrel{i}{\rightarrow} F \rightarrow L \rightarrow 0$. We claim that $i: \operatorname{im}(f) \rightarrow$ $F$ is a $\mathscr{C}$-flat preenvelope. In fact, for any $\mathscr{C}$-flat module $F_{1}$ and any homomorphism $\varphi: \operatorname{im}(f) \rightarrow F_{1}, \varphi f$ is a homomorphism from $M$ to $F_{1}$. Since $f: M \rightarrow F$ is a $\mathscr{C}$-flat preenvelope, there exists a $\psi: F \rightarrow F_{1}$ such that $\varphi f=\psi f$. Now, for any $y \in \operatorname{im}(f)$, write $y=f(x)$. Then $\varphi f(x)=\psi i f(x)$, i.e., $\varphi(y)=\psi i(y)$. It shows that $\varphi=\psi i$, and so $i: \operatorname{im}(f) \rightarrow F$ is a $\mathscr{C}$-flat preenvelope. Let $N$ be any $\mathscr{C}$-injective module. Since $R$ is left $\mathscr{C}$-coherent, $N^{+}$is $\mathscr{C}$-flat by [27, Theorem 3.3(8)], and so, the mapping $\operatorname{Hom}\left(F, N^{+}\right) \rightarrow$ $\operatorname{Hom}\left(\operatorname{im}(f), N^{+}\right)$is epic. Then, from the following commutative diagram :

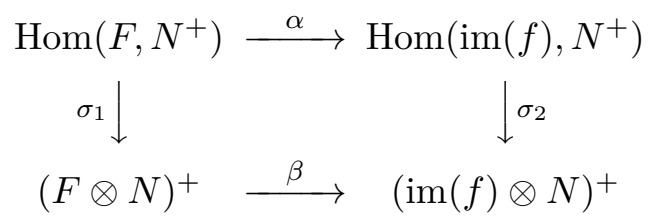

where $\sigma_{1}$ and $\sigma_{2}$ are isomorphisms, we have that the mapping $(F \otimes N)^{+} \rightarrow(\operatorname{im}(f) \otimes N)^{+}$is epic. Thus, the mapping $\operatorname{im}(f) \otimes N \rightarrow F \otimes N$ is monic. But the $\mathscr{C}$ I-flatness of $F$ implies the exactness of $0 \rightarrow \operatorname{Tor}_{1}^{R}(L, N) \rightarrow \operatorname{im}(f) \otimes N \rightarrow F \otimes N$, and therefore $\operatorname{Tor}_{1}^{R}(L, N)=0$.

\section{Strongly $\mathscr{C}$-coherent rings}

Theorem 3.1. The following statements are equivalent for a ring $R$ :

(1) $R$ is a left strongly $\mathscr{C}$-coherent ring.

(2) If $0 \rightarrow K \rightarrow E \rightarrow L \rightarrow 0$ is an exact sequence of left $R$-modules with $K \mathscr{C}$-injective and $E$ FP-injective, then $L$ is $\mathscr{C}$-injective.

(3) If $0 \rightarrow K \rightarrow E \rightarrow L \rightarrow 0$ is an exact sequence of left $R$-modules with $K \mathscr{C}$-injective and $E$ injective, then $L$ is $\mathscr{C}$-injective.

(4) $R$ is left $\mathscr{C}$-coherent, and if $0 \rightarrow N \rightarrow M \rightarrow Q \rightarrow 0$ is an exact sequence of right $R$-modules with $M$ and $Q \mathscr{C}$-flat, then $N$ is $\mathscr{C}$-flat.

(5) $R$ is left $\mathscr{C}$-coherent, and if $0 \rightarrow N \rightarrow M \rightarrow Q \rightarrow 0$ is an exact sequence of right $R$-modules with $M$ flat and $Q \mathscr{C}$-flat, then $N$ is $\mathscr{C}$-flat.

(6) $R$ is left $\mathscr{C}$-coherent, and if $0 \rightarrow N \rightarrow P \rightarrow Q \rightarrow 0$ is an exact sequence of right $R$-modules with $P$ projective and $Q \mathscr{C}$-flat, then $N$ is $\mathscr{C}$-flat.

Proof. $(1) \Rightarrow(2)$. It follows from [28, Theorem 1(7)].

$(2) \Rightarrow(3)$; and $(4) \Rightarrow(5) \Rightarrow(6)$ are trivial.

$(3) \Rightarrow(1)$. Let $M$ be a $\mathscr{C}$-injective left $R$-module. Then by (2), $E(M) / M$ is $\mathscr{C}$-injective.

And so $R$ is left strongly $\mathscr{C}$-coherent by [28, Theorem $1(8)]$.

$(1) \Rightarrow(4)$. It follows from [28, Theorem 1(9)] and [27, Proposition 3.11(2)].

$(6) \Rightarrow(1)$. For any $\mathscr{C}$-flat right $R$-module $N$, there exists an exact sequence $0 \rightarrow$ $K \rightarrow P \rightarrow N \rightarrow 0$ with $P$ projective. So $K$ is $\mathscr{C}$-flat by (6), and thus $\operatorname{Tor}_{2}^{R}(N, C) \cong$ $\operatorname{Tor}_{1}^{R}(K, C)=0$ for any $C \in \mathscr{C}$. Therefore $R$ is left strongly $\mathscr{C}$-coherent by [28, Theorem $1(11)]$.

Proposition 3.2. Let $R$ be a left strongly $\mathscr{C}$-coherent ring. Then the following statements are equivalent for a left $R$-module $M$ :

(1) $M$ is injective.

(2) $M$ is both $\mathscr{C}$-injective and $\mathscr{C}$ I-injective.

(3) There exists a $\mathscr{C}$-injective cover $f: M \rightarrow N$ with $N \mathscr{C}$ I-injective.

Proof. $(1) \Rightarrow(2)$. It is trivial.

$(2) \Rightarrow(3)$. It is clear because $M \rightarrow M$ is a $\mathscr{C}$-injective cover of M.

$(3) \Rightarrow(1)$. Consider the exact sequence $0 \rightarrow M \stackrel{i}{\rightarrow} E(M) \rightarrow E(M) / M \rightarrow 0$. Since $R$ is a left strongly $\mathscr{C}$-coherent ring, by [28, Theorem $1(7)], E(M) / M$ is $\mathscr{C}$-injective, so $\operatorname{Ext}_{R}^{1}(E(M) / M, N)=0$. Thus there exists a homomorphism $g: E(M) \rightarrow N$ such that 
$f=g i$. Since $f$ is a cover, there exists a homomorphism $h: E(M) \rightarrow M$ such that $g=f h$. Hence $f(h i)=f$, and so $h i$ is an isomorphism, this follows that $i$ is left split, and therefore $M=E(M)$ is injective.

Theorem 3.3. The following statements are equivalent for a ring $R$ :

(1) $R$ is a left strongly $\mathscr{C}$-coherent ring.

(2) $R$ is left $\mathscr{C}$-coherent, and every $\mathscr{C}$-injective $\mathscr{C}$ I-injective left $R$-module is injective.

(3) Each left $R$-module has a $\mathscr{C}$-injective cover, and every $\mathscr{C}$-injective $\mathscr{C}$ I-injective left $R$-module is injective.

(4) $R$ is left $\mathscr{C}$-coherent, and for every $\mathscr{C} I$-injective left $R$-module $L$, there there exists a $\mathscr{C}$-injective cover $E \rightarrow L$ with $E$ injective.

(5) Each left $R$-module has a $\mathscr{C}$-injective cover, and for every $\mathscr{C}$ I-injective left $R$ module $L$, there there exists a $\mathscr{C}$-injective cover $E \rightarrow L$ with $E$ injective.

(6) Every $\mathscr{C}$-pure quotient of a $\mathscr{C}$-injective left $R$-module has a $\mathscr{C}$-injective cover, and for every $\mathscr{C} I$-injective left $R$-module $L$, there exists a $\mathscr{C}$-injective cover $E \rightarrow L$ with E injective.

(7) Every $\mathscr{C}$-pure quotient of a $\mathscr{C}$-injective left $R$-module has a $\mathscr{C}$-injective cover, and every $\mathscr{C}$-injective $\mathscr{C} I$-injective left $R$-module is injective.

Proof. $(1) \Rightarrow(2)$. Since $R$ is left strongly $\mathscr{C}$-coherent, by [28, Theorem 1(10)], it is left $\mathscr{C}$-coherent. Moreover, by Proposition 3.2 , every $\mathscr{C}$-injective $\mathscr{C}$ I-injective left $R$-module is injective.

$(2) \Rightarrow(3)$. It follows from [27, Corollary 3.7].

$(1) \Rightarrow(4)$. It is clear that $R$ is left $\mathscr{C}$-coherent. Let $L$ be any $\mathscr{C}$ I-injective left $R$-module. Then by [27, Corollary 3.7], $L$ has a $\mathscr{C}$-injective cover $f: E \rightarrow L$, and by Proposition 3.2, $E$ is injective.

$(4) \Rightarrow(5)$. It follows from [27, Corollary 3.7].

$(3) \Rightarrow(7)$, and $(5) \Rightarrow(6)$ are trivial.

$(6) \Rightarrow(7)$. Let $M$ be a $\mathscr{C}$-injective $\mathscr{C}$ I-injective left $R$-module. Then by (6), there exists a $\mathscr{C}$-injective cover $f: E \rightarrow M$ with $\mathrm{E}$ injective. Note that $1_{M}: M \rightarrow M$ is also a $\mathscr{C}$-injective cover of $M$, we have that $M \cong E$, and hence $M$ is injective.

$(7) \Rightarrow(1)$. Let $0 \rightarrow N \stackrel{i}{\rightarrow} E \stackrel{f}{\rightarrow} L \rightarrow 0$ be an exact sequence of left $R$-modules with $N$ $\mathscr{C}$-injective and $E$ injective. Then by Theorem 2.6(4), this exact sequence is $\mathscr{C}$-pure, and so $L$ has a $\mathscr{C}$-injective cover $\varphi: E^{\prime} \rightarrow L$. Thus there exists a homomorphism $g: E \rightarrow E^{\prime}$ such that $f=\varphi g$. Since $f$ is epic, $\varphi$ is also epic. Now, forming a pullback we obtain the following commutative diagram with exact rows and columns (see [21, 10.3(1)]).

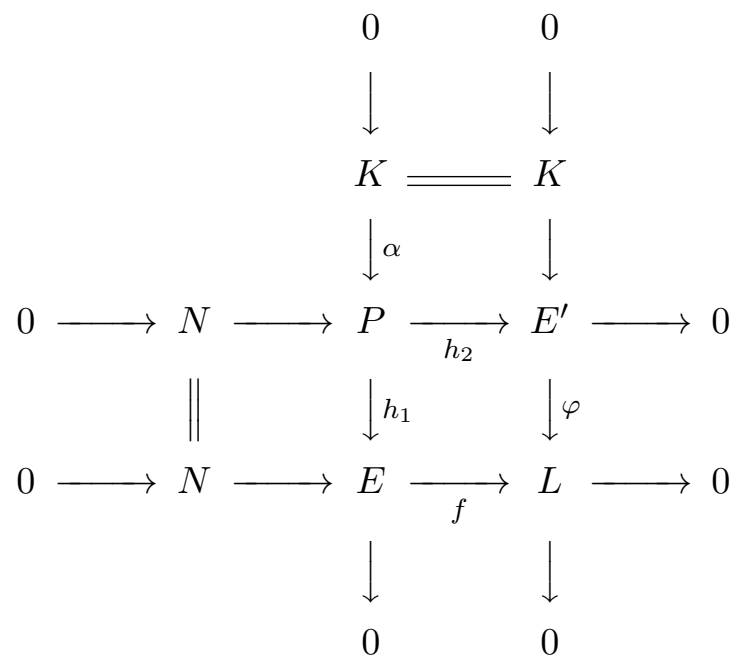


where $P=\left\{(x, y) \in E^{\prime} \oplus E \mid \varphi(x)=f(y)\right\}, K=\operatorname{Ker}(\varphi), \alpha: K \rightarrow P, k \mapsto(k, 0)$ , $h_{1}(x, y)=x, h_{2}(x, y)=y$. Let $\beta: P \rightarrow E^{\prime},(x, y) \mapsto x-g(y)$. Then $\varphi \beta(x, y)=$ $\varphi(x)-\varphi g(y)=\varphi(x)-f(y)=0$, so $\beta(x, y) \in K$, and hence $\beta$ is a homomorphism from $P$ to $K$. Note that $\beta \alpha(k)=\beta(k, 0)=k-g(0)=k$, we have that $\beta \alpha=1_{K}$. Since $N$ and $E^{\prime}$ are both $\mathscr{C}$-injective, $P$ is also $\mathscr{C}$-injective, and so $K$ is $\mathscr{C}$-injective. Note that $K$ is $\mathscr{C} \mathrm{I}$-injective by [9, Corollary 7.2.3], we have that $K$ is injective by conditions, so $L$ is $\mathscr{C}$-injective, and hence $R$ is a left strongly $\mathscr{C}$-coherent ring by Theorem 3.1(3).

Let $\mathcal{F}$ be a class of $R$-modules. According to [5], an $\mathcal{F}$-cover $\phi: F \rightarrow M$ is said to have the unique mapping property if for any homomorphism $f: F^{\prime} \rightarrow M$ with $F^{\prime} \in \mathcal{F}$, there is a unique homomorphism $g: F^{\prime} \rightarrow F$ such that $f=\phi g$.

Theorem 3.4. The following statements are equivalent for a ring $R$ :

(1) Every left $R$-module is $\mathscr{C}$-projective.

(2) Every nonzero left $R$-module has a nonzero $\mathscr{C}$-projective submodule.

(3) $R$ is left strongly $\mathscr{C}$-coherent, and every $(\mathscr{C}$-injective) left $R$-module has a $\mathscr{C}$ projective cover with the unique mapping property.

Proof. $(1) \Rightarrow(2)$ and $(1) \Rightarrow(3)$ are obvious.

$(2) \Rightarrow(1)$. Assume $(2)$. To prove $(1)$, we need only to prove that every $\mathscr{C}$-injective module $E$ is injective by [28, Theorem $6(3)]$.

Let $I$ be a left ideal of $R, i: I \rightarrow R$ be the inclusion map and $f: I \rightarrow E$ be any homomorphism. It suffices to show that there is $g: R \rightarrow E$ that extends $f$. Let $\mathscr{A}$ consist of all pair $\left(I^{\prime}, g^{\prime}\right)$, where $I \subseteq I^{\prime} \subseteq R$ and $g^{\prime}: I^{\prime} \rightarrow E$ extends $f$. Since $(I, f) \in \mathscr{A}, \mathscr{A} \neq \phi$. $\mathscr{A}$ is a partially set by saying $\left(I^{\prime}, g^{\prime}\right) \leq\left(I^{\prime \prime}, g^{\prime \prime}\right)$ if $I^{\prime} \subseteq I^{\prime \prime}$ and $g^{\prime \prime}$ extends $g^{\prime}$. By Zorn's Lemma, there is a maximal element $\left(I_{0}, g_{0}\right)$ in $\mathscr{A}$. If $I_{0} \neq R$, then $R / I_{0} \neq 0$. By (2), there is a nonzero $\mathscr{C}$-projective submodule $K / I_{0}$ of $R / I_{0}$. Note that $\operatorname{Ext}_{R}^{1}\left(K / I_{0}, E\right)=0$, we have that $g_{0}$ can be extended to $K$, this contradicts to the maximality of $\left(I_{0}, g_{0}\right)$. Thus, $I_{0}=R$ and $E$ is injective, as required.

$(3) \Rightarrow(1)$. Assume (3). To prove (1), we need only to prove that every $\mathscr{C}$-injective module $E$ is $\mathscr{C}$-projective by [28, Theorem 6(4)]. By (3), $E$ has a $\mathscr{C}$-projective cover $\phi: P \rightarrow E$ with the unique mapping property. Let $K=\operatorname{Ker}(\phi), i: K \rightarrow P$ be the inclusion map and $\varphi: P^{\prime} \rightarrow K$ be a $\mathscr{C}$-projective cover of $K$. Then $\phi i \varphi=0=\phi 0$, and so $i \varphi=0$ by the unique mapping property. Since every $\mathscr{C}$-projective cover is epic, $\varphi$ and $\phi$ are epic, so $\phi$ is an isomorphism, and thus $E$ is $\mathscr{C}$-projective. This completes the proof.

According to [28], the $\mathscr{C}$-injective dimension of a module ${ }_{R} M$ is defined by

$$
\mathscr{C} \mathrm{J}-\operatorname{dim}\left({ }_{R} M\right)=\inf \left\{n: \operatorname{Ext}_{R}^{n+1}(C, M)=0 \text { for every } C \in \mathscr{C}\right\}
$$

the $\mathscr{C}$-injective global dimension of a ring $R$ is defined by

$\mathscr{C} \mathrm{J}-\mathrm{GLD}(\mathrm{R})=\sup \{\mathscr{C} \mathrm{J}-\operatorname{dim}(M): M$ is a left $R$-module $\}$;

the $\mathscr{C}$-flat dimension of a module $M_{R}$ is defined by

$$
\mathscr{C} \mathcal{F}-\operatorname{dim}\left(M_{R}\right)=\inf \left\{n: \operatorname{Tor}_{n+1}^{R}(M, C)=0 \text { for every } C \in \mathscr{C}\right\}
$$

the $\mathscr{C}$-weak global dimension of a ring $R$ is defined by

$\mathscr{C}-\mathrm{WD}(\mathrm{R})=\sup \{\mathscr{C} \mathcal{F}-\operatorname{dim}(M): M$ is a right $R$-module $\}$.

Theorem 3.5. Let $R$ be a left strongly $\mathscr{C}$-coherent ring, $M$ a left $R$-module and $n$ a nonnegative integer. Then the following statements are equivalent:

(1) $\mathscr{C} \mathcal{J}-\operatorname{dim}\left({ }_{R} M\right) \leq n$.

(2) $\operatorname{Ext}_{R}^{n+k}(P, M)=0$ for all $\mathscr{C}$-projective module $P$ and all positive integers $k$.

(3) $\operatorname{Ext}_{R}^{n+1}(P, M)=0$ for all $\mathscr{C}$-projective module $P$. 
Proof. $(1) \Rightarrow(2)$. Assume (1). Then since $R$ is left strongly $\mathscr{C}$-coherent, by [28, Theorem 2], there exists an exact sequence of left $R$-modules $0 \rightarrow M \stackrel{\varepsilon}{\rightarrow} E_{0} \stackrel{d_{0}}{\rightarrow} \cdots \rightarrow$ $E_{n-1} \stackrel{d_{n-1}}{\rightarrow} E_{n} \rightarrow 0$ such that $E_{0}, \cdots, E_{n-1}, E_{n}$ are $\mathscr{C}$-injective. Thus, by [28, Theorem 1(12)], we have $\operatorname{Ext}_{R}^{n+1}(P, M) \cong \operatorname{Ext}_{R}^{n}\left(P, i m\left(d_{0}\right)\right) \cong \operatorname{Ext}_{R}^{n-1}\left(P, i m\left(d_{1}\right)\right) \cong \cdots \cong$ $\operatorname{Ext}_{R}^{1}\left(P, i m\left(d_{n-1}\right)\right)=\operatorname{Ext}_{R}^{1}\left(P, E_{n}\right)=0$ for any $\mathscr{C}$-projective module $P$, and $\operatorname{Ext}_{R}^{n+k}(P, M) \cong$ $\operatorname{Ext}_{R}^{1}(P, 0)=0$ for any $k>1$. So (2) follows.

$(2) \Rightarrow(3) \Rightarrow(1)$. It is trivial.

Corollary 3.6. Let $R$ be a left strongly $\mathscr{C}$-coherent ring and $0 \rightarrow A \rightarrow B \rightarrow C \rightarrow 0$ an exact sequence of left $R$-modules. If two of $\mathscr{C} \mathrm{J}$-dim $(A), \mathscr{C} \mathrm{J}-\operatorname{dim}(B), \mathscr{C} \mathrm{J}$-dim $(C)$ are finite, then so is the third. Moreover:

(1) $\mathscr{C} \mathrm{J}-\operatorname{dim}(B) \leq \sup \{\mathscr{C} \mathrm{J}-\operatorname{dim}(A), \mathscr{C} \mathrm{J}-\operatorname{dim}(C)\}$.

(2) $\mathscr{C} J-d i m(A) \leq \sup \{\mathscr{C} \mathrm{J}-\operatorname{dim}(B), \mathscr{C} \mathrm{J}-\operatorname{dim}(C)+1\}$.

(3) $\mathscr{C} \mathrm{J}-\operatorname{dim}(C) \leq \sup \{\mathscr{C} \mathrm{J}-\operatorname{dim}(B), \mathscr{C} \mathrm{J}-\operatorname{dim}(A)-1\}$. In particular, $\mathscr{C} \mathrm{J}-\operatorname{dim}(A \oplus C)=\sup \{\mathscr{C} \mathrm{J}-\operatorname{dim}(A), \mathscr{C} \mathrm{J}-\operatorname{dim}(C)\}$.

Let $n$ be a positive integer. then according to [4], a left $R$-module $M$ is said to be $n$-presented in case there is an exact sequence of left $R$-modules $F_{n} \rightarrow F_{n-1} \rightarrow \cdots \rightarrow$ $F_{1} \rightarrow F_{0} \rightarrow M \rightarrow 0$ in which every $F_{i}$ is finitely generated free. It is easy to see that a left $R$-module $M$ is $n$-presented if and only if there exists an exact sequence of left $R$-modules $0 \rightarrow K_{n} \rightarrow F_{n-1} \rightarrow \cdots \rightarrow F_{1} \rightarrow F_{0} \rightarrow M \rightarrow 0$ such that $F_{0}, \cdots, F_{n-1}$ are finitely generated free and $K_{n}$ is finitely generated.

Lemma 3.7. Let $R$ be a left strongly $\mathscr{C}$-coherent ring. Then every $C \in \mathscr{C}$ is n-presented for any positive integer $n$.

Proof. Use induction on $n$. If $n=1$, then it is clear that the result holds. Assume that every $C \in \mathscr{C}$ is $n$-presented. Then for any $C \in \mathscr{C}$ and any FP-injective module $N$, we have $\operatorname{Ext}_{R}^{n+1}(C, N)=0$ by [28, Theorem $\left.1(5)\right]$ because $R$ is left strongly $\mathscr{C}$-coherent. Let $0 \rightarrow K_{n} \rightarrow F_{n-1} \rightarrow \cdots \rightarrow F_{1} \rightarrow F_{0} \rightarrow C \rightarrow 0$ be an exact sequence of left $R$-modules with $F_{0}, \cdots, F_{n-1}$ finitely generated free left $\mathrm{R}$-modules and $K_{n}$ finitely generated. Then $\operatorname{Ext}_{R}^{1}\left(K_{n}, N\right) \cong \operatorname{Ext}_{R}^{n+1}(C, N)=0$, so $K_{n}$ is finitely presented by [7], and hence $C$ is $(n+1)$-presented.

Theorem 3.8. Let $R$ be a left strongly $\mathscr{C}$-coherent ring and $M$ a left $R$-module. Then $\mathscr{C} \mathrm{J}-\operatorname{dim}(M)=\mathscr{C} \mathcal{F}-\operatorname{dim}\left(M^{+}\right)$.

Proof. Let $n$ be a positive integer, $C \in \mathscr{C}$. Since $R$ is left strongly $\mathscr{C}$-coherent, by Lemma 3.7, $C$ is $(n+2)$-presented. So, by [2, Lemma 2.7(2)], we have $\operatorname{Tor}_{n+1}^{R}\left(M^{+}, C\right) \cong$ $\operatorname{Ext}_{R}^{n+1}(C, M)^{+}$. Consequently, $\mathscr{C} \mathcal{J}-\operatorname{dim}(M)=\mathscr{C} \mathcal{F}-\operatorname{dim}\left(M^{+}\right)$by $[28$, Theorem 2, Theorem 3].

Theorem 3.9. Let $R$ be left strongly $\mathscr{C}$-coherent and ${ }_{R} R$ be $\mathscr{C}$-injective. If ${ }_{R} M$ is $\mathscr{C}$ projective with finite projective dimension, then ${ }_{R} M$ is projective.

Proof. Suppose that ${ }_{R} M$ is $\mathscr{C}$-projective with $p d(M)=n<\infty$. Then by [28, Theorem $5]$, there exists an exact sequence of left $R$-modules

$$
0 \rightarrow P_{n} \stackrel{d_{n}}{\rightarrow} P_{n-1} \stackrel{d_{n-1}}{\rightarrow} \cdots \rightarrow P_{1} \stackrel{d_{1}}{\rightarrow} P_{0} \stackrel{d_{0}}{\rightarrow} M \rightarrow 0
$$

such that $P_{0}, \cdots, P_{n-1}, P_{n}$ are projective. Since ${ }_{R} R$ is $\mathscr{C}$-injective and direct sums and direct summands of $\mathscr{C}$-injective modules are $\mathscr{C}$-injective by [28, Proposition 2.5], each $P_{i}$ is $\mathscr{C}$-injective for $i=0,1, \cdots, n$. Clearly, $i m\left(d_{n}\right) \cong P_{n}$ is $\mathscr{C}$-injective. Note that $R$ is left strongly $\mathscr{C}$-coherent, by [28, Theorem $1(7)], i m\left(d_{n-1}\right)$ is $\mathscr{C}$-injective. Continues in this way, one can get that $i m\left(d_{1}\right)$ is $\mathscr{C}$-injective, so $\operatorname{Ext}_{R}^{1}\left(M, i m\left(d_{1}\right)\right)=0$, and thus the exact sequence $0 \rightarrow i m\left(d_{1}\right) \rightarrow P_{0} \rightarrow M \rightarrow 0$ is split, this follows that ${ }_{R} M$ is projective, as required. 
Recall that, by [28, Example 1], a left $\mathscr{C}$-coherent ring need not be left strongly $\mathscr{C}$ coherent. As the end of this section, we give another example which shows that even if $R$ is a left artinian ring, it need not be left strongly $\mathscr{C}$-coherent.

Example 3.10. Let $K$ be a field and $L$ be a proper subfield of $K$ such that $\rho: K \rightarrow L$ is an isomorphism. Let $K[x ; \rho]$ be the ring of twisted right polynomials over $K$ where $k x=x \rho(k)$ for all $k \in K$. Set $R=K[x ; \rho] /\left(x^{2}\right)$, and $\mathscr{C}=\{R / R a: a \in R\}$. If $b_{1}, b_{2}$ is a basis for $K$ as a vector space over $L$, then $R$ is left artinian and hence left $\mathscr{C}$-coherent, but it is not left strongly $\mathscr{C}$-coherent.

Proof. Since $K$ has finite vector space dimension over $L$, by [18, Example 1], $R$ is left artinian . Since the only proper right ideal of $R$ is $\mathbf{r}_{R}(x)=x R=x K$, it is readily verified that $\mathbf{r}_{R} \mathbf{l}_{R}(a)=a R$ for any $a \in R$, so $R R$ is P-injective by [16, Lemma 1.1]. Now, we define $f: R x b_{1}+R x b_{2} \rightarrow R$ by $f\left(r_{1} x b_{1}+r_{2} x b_{2}\right)=r_{1} x+r_{2} x$, then it is easy to see that $f$ is a left $R$-homomorphism. We claim that this homomorphism can not be extended to an endomorphism of $R$. Otherwise, there exists a $c=k_{0}+x k_{0}^{\prime} \in R$ such that $f=\cdot c$. Clearly, $k_{0} \neq 0$. Thus, $f\left(x b_{1}-x b_{2}\right)=\left(x b_{1}-x b_{2}\right)\left(k_{0}+x k_{0}^{\prime}\right)$, and so $0=x-x=\left(x b_{1}-x b_{2}\right) k_{0}$, this follows that $b_{1}=b_{2}$, a contradiction. Observing that $\mathbf{l}_{R}(x)=x K=x R=R x b_{1}+R x b_{2}$, we have $\operatorname{Ext}_{R}^{1}(R x, R) \cong \operatorname{Ext}_{R}^{1}\left(R /\left(R x b_{1}+R x b_{2}\right), R\right) \neq 0$, and hence $R$ is not left strongly $\mathscr{C}$-coherent.

\section{4. $\mathscr{C}$-semihereditary rings}

We begin with the following definition.

Definition 4.1. A ring $\mathrm{R}$ is called weakly $\mathscr{C}$-semihereditary, if whenever $0 \rightarrow K \rightarrow P \rightarrow$ $C \rightarrow 0$ is exact, where $C \in \mathscr{C}, \mathrm{P}$ is finitely generated projective, then $K$ is flat.

Recall that a ring $R$ is called left weakly $n$-semihereditary [25] if every $n$-generated left ideal is flat; a ring $R$ is called a left p.f ring [11] if every principal left ideal of $R$ is flat. By [11, Theorem 2.2], a ring $R$ is left p.f if and only if it is right p.f; a ring $R$ is called a left FS-ring $[12,22]$ if $\operatorname{Soc}\left({ }_{R} R\right)$ is flat.

Example 4.2. (1). Let $\mathscr{C}=\{R / I: I$ is an $n$-generated left ideal of $R\}$. Then the ring $R$ is weakly $\mathscr{C}$-semihereditary if and only if $R$ is left weakly $n$-semihereditary.

(2). Let $\mathscr{C}=\{R / R a: a \in R\}$. Then the $\operatorname{ring} R$ is weakly $\mathscr{C}$-semihereditary if and only if $R$ is left p.f.

(3). Let $\mathscr{C}=\{R / R a: R a$ is a minimal left ideal of $R\}$. Then the ring $R$ is weakly $\mathscr{C}$-semihereditary if and only if every minimal left ideal of $R$ is flat, if and only if $R$ is a left FS-ring .

Theorem 4.3. The following statements are equivalent for a ring $R$ :

(1) $R$ is a left weakly $\mathscr{C}$-semihereditary ring.

(2) Every submodule of a $\mathscr{C}$-flat right $R$-module is $\mathscr{C}$-flat.

(3) Every submodule of a flat right $R$-module is $\mathscr{C}$-flat.

(4) Every submodule of a projective right $R$-module is $\mathscr{C}$-flat.

(5) Every submodule of a free right $R$-module is $\mathscr{C}$-flat.

(6) Every finitely generated right ideal of $R$ is $\mathscr{C}$-flat.

Proof. $(2) \Rightarrow(3) \Rightarrow(4) \Rightarrow(5) \Rightarrow(6)$ is trivial.

$(1) \Rightarrow(2)$. Assume (1). Let $A$ be a submodule of a $\mathscr{C}$-flat right $R$-module $B$ and let $C \in \mathscr{C}$. Then there exists an exact sequence of left $R$-modules $0 \rightarrow K \rightarrow P \rightarrow C \rightarrow$ 0 , where $P$ is finitely generated projective. By (1), $K$ is flat. Then the exactness of $0=\operatorname{Tor}_{2}^{R}(B / A, P) \rightarrow \operatorname{Tor}_{2}^{R}(B / A, C) \rightarrow \operatorname{Tor}_{1}^{R}(B / A, K)=0$ implies that $\operatorname{Tor}_{2}^{R}(B / A, C)=$ 0 . And thus from the exactness of the sequence $0=\operatorname{Tor}_{2}^{R}(B / A, C) \rightarrow \operatorname{Tor}_{1}^{R}(A, C) \rightarrow$ $\operatorname{Tor}_{1}^{R}(B, C)=0$ we have $\operatorname{Tor}_{1}^{R}(A, C)=0$. It shows that $A$ is $\mathscr{C}$-flat. 
$(6) \Rightarrow(1)$. Let $C \in \mathscr{C}$. There exists an exact sequence of left $R$-modules $0 \rightarrow K \rightarrow$ $P \rightarrow C \rightarrow 0$, where $P$ is finitely generated projective. For any finitely generated right ideal $I$ of $R$, we have an exact sequence $0 \rightarrow \operatorname{Tor}_{2}^{R}(R / I, C) \rightarrow \operatorname{Tor}_{1}^{R}(I, C)=0$ since $I$ is $\mathscr{C}$-flat. So $\operatorname{Tor}_{2}^{R}(R / I, C)=0$, and hence we obtain an exact sequence $0=\operatorname{Tor}_{2}^{R}(R / I, C) \rightarrow$ $\operatorname{Tor}_{1}^{R}(R / I, K) \rightarrow 0$. Thus, $\operatorname{Tor}_{1}^{R}(R / I, K)=0$. And so $K$ is flat.

Proposition 4.4. If $R$ is a left weakly $\mathscr{C}$-semihereditary ring, then $\mathscr{C}-W D(R) \leq 1$.

Proof. Let $M$ be any right $R$-module and let $C \in \mathscr{C}$. Then there exists an exact sequence of left $R$-modules $0 \rightarrow K \rightarrow P \rightarrow C \rightarrow 0$, where $P$ is finitely generated projective. Since $R$ is left weakly $\mathscr{C}$-semihereditary, $K$ is flat. $\operatorname{So~}_{\operatorname{Tor}_{2}^{R}}^{R}(M, C) \cong \operatorname{Tor}_{1}^{R}(M, K)=0$. It shows that $\mathscr{C}-\mathrm{WD}(\mathrm{R}) \leq 1$.

Lemma 4.5. Let $\mathcal{F}$ be a class of some right $R$-modules. If $N \stackrel{f_{1}}{\rightarrow} N_{1}$ and $N \stackrel{f_{2}}{\rightarrow} N_{2}$ are $\mathcal{F}$-preenvelopes, then $N_{1} \oplus N_{2} / f_{2}(N) \cong N_{2} \oplus N_{1} / f_{1}(N)$.

Proof. Let $\varepsilon_{i}: N_{i} \rightarrow N_{1} \oplus N_{2}$ be the injections, $i=1,2$. We obtain a morphism $q^{*}=\varepsilon_{1} f_{1}+\varepsilon_{2} f_{2}: N \rightarrow N_{1} \oplus N_{2}$. Let $\overline{\varepsilon_{1}}: N_{1} \rightarrow \operatorname{Coker}\left(q^{*}\right) ; n_{1} \mapsto\left(n_{1}, 0\right)+i m\left(q^{*}\right)$ , $\overline{\varepsilon_{2}}: N_{2} \rightarrow \operatorname{Coker}\left(q^{*}\right) ; n_{2} \mapsto\left(0, n_{2}\right)+i m\left(q^{*}\right)$ and $Q=\operatorname{Coker}\left(q^{*}\right)$. Then we get the following pushout diagram:

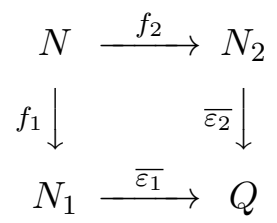

And so, by the proof of $[21,10.6(1)(\mathrm{i})]$, we have the following commutative diagram with exact rows, where $g: Q \rightarrow N_{2} / f_{2}(N) ;\left(n_{1}, n_{2}\right)+i m\left(q^{*}\right) \mapsto n_{2}+f_{2}(N)$ :

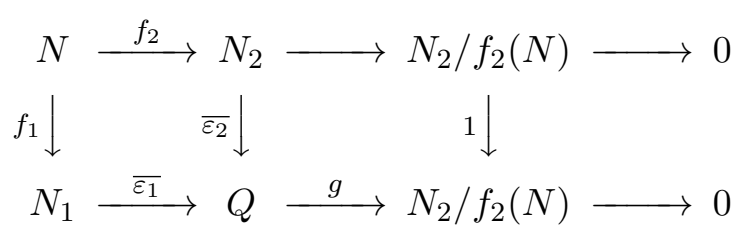

Since $N \stackrel{f_{2}}{\rightarrow} N_{2}$ is an $\mathcal{F}$-preenvelope and $N_{1} \in \mathcal{F}$, there exists a homomorphism $\alpha: N_{2} \rightarrow N_{1}$ such that $f_{1}=\alpha f_{2}$. If $\bar{\varepsilon}_{1}\left(n_{1}\right)=0$, then $\left(n_{1}, 0\right)=q^{*}(n)=\left(f_{1}(n), f_{2}(n)\right)$ for some $n \in N$, so $f_{2}(n)=0, f_{1}(n)=n_{1}$, and hence $n_{1}=f_{1}(n)=\alpha f_{2}(n)=0$. It shows that $\overline{\varepsilon_{1}}$ is monic. Now, we define $h: Q \rightarrow N_{1}$ by $\left(n_{1}, n_{2}\right)+i m\left(q^{*}\right) \mapsto n_{1}-\alpha\left(n_{2}\right)$. Then $h$ is well-defined, and $h \overline{\varepsilon_{1}}\left(n_{1}\right)=h\left(\left(n_{1}, 0\right)+i m\left(q^{*}\right)\right)=n_{1}-\alpha(0)=n_{1}$ for each $n_{1} \in N_{1}$, so $h \overline{\varepsilon_{1}}=1_{N_{1}}$, and then $\overline{\varepsilon_{1}}$ is left split. Thus, we have $Q \cong N_{1} \oplus N_{2} / f_{2}(N)$. Similarly, we have also that $Q \cong N_{2} \oplus N_{1} / f_{1}(N)$ and so $N_{1} \oplus N_{2} / f_{2}(N) \cong N_{2} \oplus N_{1} / f_{1}(N)$.

Next, we give some new characterizations of left $\mathscr{C}$-semihereditary rings.

Theorem 4.6. The following statements are equivalent for a ring $R$ :

(1) $R$ is left $\mathscr{C}$-semihereditary.

(2) $R$ is left $\mathscr{C}$-coherent and left weakly $\mathscr{C}$-semihereditary.

(3) $R$ is left strongly $\mathscr{C}$-coherent and every $\mathscr{C}$-projective left $R$-module has a monic $\mathscr{C}$-injective cover.

(4) Every $\mathscr{C}$-projective left $R$-module has projective dimension at most 1.

(5) $R$ is left $\mathscr{C}$-coherent and every $\mathscr{C}$ I-injective module is injective.

(6) Every left $R$-module has a $\mathscr{C}$-injective cover and every $\mathscr{C}$ I-injective module is injective.

(7) Every $\mathscr{C}$-pure quotient of a $\mathscr{C}$-injective left $R$-module has a $\mathscr{C}$-injective cover and every $\mathscr{C}$ I-injective module is injective.

(8) $R$ is left strongly $\mathscr{C}$-coherent and every $\mathscr{C}$ I-injective module is $\mathscr{C}$-injective. 
(9) $R$ is left strongly $\mathscr{C}$-coherent and the kernel of any $\mathscr{C}$-injective precover of a left $R$-module is $\mathscr{C}$-injective.

(10) $R$ is left strongly $\mathscr{C}$-coherent and the kernel of any $\mathscr{C}$-injective cover of a left $R$-module is $\mathscr{C}$-injective.

(11) $R$ is left strongly $\mathscr{C}$-coherent and the cokernel of any $\mathscr{C}$-injective preenvelope of a left $R$-module is $\mathscr{C}$-injective.

(12) $R$ is left strongly $\mathscr{C}$-coherent and the kernel of any $\mathscr{C}$-flat precover of a right $R$ module is $\mathscr{C}$-flat.

(13) $R$ is left strongly $\mathscr{C}$-coherent and the kernel of any $\mathscr{C}$-flat cover of a right $R$-module is $\mathscr{C}$-flat.

(14) $R$ is left strongly $\mathscr{C}$-coherent and the cokernel of any $\mathscr{C}$-flat preenvelope of a right $R$-module is $\mathscr{C}$-flat.

Proof. $(1) \Leftrightarrow(2)$. It follows from [27, Theorem 4.3(2)] and Theorem 4.3(2).

$(1) \Rightarrow(3)$. Suppose that $R$ is left $\mathscr{C}$-semihereditary. Then it is left strongly $\mathscr{C}$-coherent by [28, Theorem 4]. Moreover, by [27, Theorem 4.3(7)], every $\mathscr{C}$-projective left $R$-module has a monic $\mathscr{C}$-injective cover.

$(3) \Rightarrow(1)$. Let $E$ be any injective left $R$-module and $K$ any submodule of $E$. By [27, Theorem $4.3(6)]$, we need only to prove that $E / K$ is $\mathscr{C}$-injective. In fact, since $(\mathscr{C} \mathcal{P}, \mathscr{C} J)$ is a complete cotorsion pair by [27, Theorem 2.10(1)], there exists an exact sequences $0 \rightarrow K \rightarrow E_{1} \stackrel{f}{\rightarrow} P \rightarrow 0$ with $P \mathscr{C}$-projective and $E_{1} \mathscr{C}$-injective. By (3), $P$ has a monic $\mathscr{C}$-injective cover $\varphi: E_{2} \rightarrow P$. So, there exists a homomorphism $g: E_{1} \rightarrow E_{2}$ such that $f=\varphi g$. Thus $\varphi$ is epic, and hence $\varphi$ is an isomorphism. This implies that $P$ is $\mathscr{C}$-injective. For any $C \in \mathscr{C}$, we have the exact sequence

$$
0=\operatorname{Ext}_{R}^{1}(C, P) \rightarrow \operatorname{Ext}_{R}^{2}(C, K) \rightarrow \operatorname{Ext}_{R}^{2}\left(C, E_{1}\right) .
$$

But $R$ is left strongly $\mathscr{C}$-coherent, by [28, Theorem 1(6)], $\operatorname{Ext}_{R}^{2}\left(C, E_{1}\right)=0$, and so $\operatorname{Ext}_{R}^{2}(C, K)=0$. On the other hand, the short exact sequence $0 \rightarrow K \rightarrow E \rightarrow E / K \rightarrow 0$ induces the exact sequence

$$
0=\operatorname{Ext}_{R}^{1}(C, E) \rightarrow \operatorname{Ext}_{R}^{1}(C, E / K) \rightarrow \operatorname{Ext}_{R}^{2}(C, K)=0 .
$$

so, we have $\operatorname{Ext}_{R}^{1}(C, E / K)=0$, and hence $E / K$ is $\mathscr{C}$-injective. Consequently, $R$ is left $\mathscr{C}$-semihereditary by $[27$, Theorem $4.3(6)]$.

$(1) \Rightarrow(4)$. Let $M$ be a $\mathscr{C}$-projective module and $N$ be any left $R$-module. Since $R$ is left $\mathscr{C}$-semihereditary, by [27, Theorem $4.3(6)], E(N) / N$ is $\mathscr{C}$-injective. So, by the exactness of the sequence

$$
0=\operatorname{Ext}_{R}^{1}(M, E(N) / N) \rightarrow \operatorname{Ext}_{R}^{2}(M, N) \rightarrow \operatorname{Ext}_{R}^{2}(M, E(N))=0 .
$$

We have $\operatorname{Ext}_{R}^{2}(M, N)=0$, and hence $M$ has projective dimension at most 1 .

(4) $\Rightarrow(1)$. Let $C \in \mathscr{C}$ and $0 \rightarrow K \rightarrow P \rightarrow C \rightarrow 0$ be exact, where $P$ is finitely generated projective. Note that $C$ is $\mathscr{C}$-projective, by $(4), p d(C) \leq 1$, and so $K$ is projective by Schanuel's Lemma.

$(1) \Rightarrow(5)$. Since $R$ is left $\mathscr{C}$-semihereditary, by [27, Theorem 4.3], $R$ is left $\mathscr{C}$-coherent and every quotient module of an injective left $R$-module is $\mathscr{C}$-injective . Let $M$ be a $\mathscr{C}$ I-injective left $R$-module. Then $E(M) / M$ is $\mathscr{C}$-injective, so $M$ is injective with respect to the exact sequence $0 \rightarrow M \rightarrow E(M) \rightarrow E(M) / M \rightarrow 0$ by Proposition 2.3, and hence $M=E(M)$ is injective.

$(5) \Rightarrow(6)$. It follows from [27, Corollary 3.7].

$(6) \Rightarrow(1)$. Let $M$ be a quotient of an injective left $R$-module. By (6), $M$ has a $\mathscr{C}$ injective cover. Suppose $f: F \rightarrow M$ is a $\mathscr{C}$-injective cover of $M$. Then $f$ is epic. By Remark 2.4, $\operatorname{Ker}(f)$ is $\mathscr{C}$ I-injective, and so it is injective by (6). Thus, $M$ is isomorphic to a direct summand of $F$ and hence it is $\mathscr{C}$-injective. Hence, by [27, Theorem 4.3(6)], $R$ is left $\mathscr{C}$-semihereditary. 
$(6) \Rightarrow(7)$. It is obvious.

$(7) \Rightarrow(8)$. It follows from Theorem $3.3(7)$.

$(8) \Rightarrow(5)$. Assume $(8)$. Then by $[28$, Theorem $1(10)], R$ is left $\mathscr{C}$-coherent. Let $M$ be a $\mathscr{C}$ I-injective module. Then by (8), $M$ is $\mathscr{C}$-injective. But $R$ is left strongly $\mathscr{C}$-coherent, by [28, Theorem 1(7)], $E(M) / M$ is $\mathscr{C}$-injective. Thus, by Proposition 2.3(4), $M$ is injective .

$(1) \Rightarrow(9)$. Clearly, $R$ is left strongly $\mathscr{C}$-coherent . Let $f: F \rightarrow M$ be a $\mathscr{C}$-injective precover and $K=\operatorname{Ker}(f)$. Since $R$ is left $\mathscr{C}$-semihereditary, by [27, Theorem 4.3(7)], there exists a monic $\mathscr{C}$-injective cover $\varphi: G \rightarrow M$. Thus, by [9, Lemma 8.6.3], we have $K \oplus G \cong F$, and so $K$ is $\mathscr{C}$-injective.

$(9) \Rightarrow(10)$. It is obvious.

$(10) \Rightarrow(1)$. Let $M$ be a quotient of a $\mathscr{C}$-injective left $R$-module. Since $R$ is left $\mathscr{C}$ coherent, by [27, Corollary 3.7], $M$ has a $\mathscr{C}$-injective cover $f: F \rightarrow M$. Clearly, $f$ is epic. So, by (10), we have that $\operatorname{Ker}(f)$ is $\mathscr{C}$-injective, this implies that $M$ is also $\mathscr{C}$-injective by [28, Theorem $1(7)]$ as $R$ is left strongly $\mathscr{C}$-coherent. Therefore, by [27, Theorem 4.3(5)], $R$ is left $\mathscr{C}$-semihereditary.

$(1) \Rightarrow(11)$. Clearly, $R$ is left strongly $\mathscr{C}$-coherent. And by [27, Theorem $4.3(5)]$, every quotient module of a $\mathscr{C}$-injective module is $\mathscr{C}$-injective, so the cokernel of any $\mathscr{C}$-injective preenvelope of a left $R$-module is $\mathscr{C}$-injective.

$(11) \Rightarrow(1)$. Let $M$ be any left $R$-module. Since the class of all $\mathscr{C}$-injective left $R$-modules is closed under pure submodules, isomorphisms and direct product, by [29, Theorem 2.6], $M$ has a $\mathscr{C}$-injective preenvelope $f: M \rightarrow E$. By $(11), E / i m(f)$ is $\mathscr{C}$-injective . It is easy to see that $f$ is monic. Since $R$ is left strongly $\mathscr{C}$-coherent, by [28, Theorem 2(5)], $\mathscr{C}$ J- $\operatorname{dim}\left({ }_{R} M\right) \leq 1$. And so, $\mathscr{C}$ J-GLD $(\mathrm{R}) \leq 1$. Therefore, by [28, Theorem $\left.4(2)\right], R$ is left $\mathscr{C}$-semihereditary.

$(1) \Rightarrow(12)$. Clearly, $R$ is left strongly $\mathscr{C}$-coherent. And by [27, Theorem $4.3(2)]$, the kernel of any $\mathscr{C}$-flat precover of a right $R$-module is $\mathscr{C}$-flat.

$(12) \Rightarrow(13)$. It is obvious.

$(13) \Rightarrow(1)$. Let $N$ be any right $R$-module. Then by $[27$, Theorem $2.10(2)], N$ has a $\mathscr{C}$ flat cover $f: F \rightarrow N$. Clearly, $f$ is epic. By (13), we have that $\operatorname{Ker}(f)$ is $\mathscr{C}$-flat. But $R$ is left strongly $\mathscr{C}$-coherent, by $[28$, Theorem $3(5)], \mathscr{C} \mathcal{F}$ - $\operatorname{dim}\left(N_{R}\right) \leq 1$. Thus, $\mathscr{C}-\mathrm{WD}(\mathrm{R}) \leq 1$. Consequently, by [28, Theorem 4(3)], we have that $R$ is left $\mathscr{C}$-semihereditary.

$(1) \Rightarrow(14)$. Clearly, $R$ is left strongly $\mathscr{C}$-coherent. Let $\varphi: N \rightarrow F$ be a $\mathscr{C}$-flat preenvelope of a right $R$-module $N$ and $L=\operatorname{coker}(\varphi)$. Since $R$ is left $\mathscr{C}$-semihereditary, by [27, Theorem 4.3(8)], $N$ has an epic $\mathscr{C}$-flat envelope $\phi: N \rightarrow G$. Hence, by Lemma 4.5, we have $F \cong G \oplus L$, and so $L$ is $\mathscr{C}$-flat.

$(14) \Rightarrow(1)$. Let $N$ be a submodule of a $\mathscr{C}$-flat module. Since $R$ is left $\mathscr{C}$-coherent, by [27, Theorem 3.3(12)], $N$ has a $\mathscr{C}$-flat preenvelope $f: N \rightarrow F$. It is easy to see that $f$ is monic. By (14), $F / i m(f)$ is $\mathscr{C}$-flat. Note that $R$ is left strongly $\mathscr{C}$-coherent, by Theorem $3.1(4), N$ is $\mathscr{C}$-flat. Therefore, by [27, Theorem 4.3(2)], $R$ is left $\mathscr{C}$-semihereditary.

Acknowledgment. The authors would like to thank the referee for the useful suggestions and comments. This research was supported by the Natural Science Foundation of Zhejiang Province, China(LY18A010018).

\section{References}

[1] S. U. Chase, Direct products of modules, Trans. Amer. Math. Soc. 97 , 457-473, 1960.

[2] J. L. Chen and N. Q. Ding, On n-coherent rings, Comm. Algebra 24 (3), 3211-3216, 1996.

[3] R. R. Colby, Rings which have flat injective modules, J. Algebra 35, 239-252, 1975.

[4] D. L. Costa, Parameterizing families of non-noetherian rings, Comm. Algebra 22 (10), 3997-4011, 1994. 
[5] N. Q. Ding and J. L. Chen, On envelopes with the unique mapping property, Comm. Algebra 24 (4), 1459-1470, 1996.

[6] D. E. Dobbs, S. Kabbaj, and N. Mahdou, n-coherent rings and modules, Lecture Notes in Pure and Appl. Math. 185, 269-281, 1997

[7] E. Enochs, A note on absolutely pure modules, Canad. Math. Bull. 19 (3), 361-362, 1976.

[8] E. E. Enochs and O. M. G. Jenda, Copure injective resolutions, flat resolutions and dimensions, Comment. Math. Univ. Carolin 34 (2), 203-211, 1993.

[9] E. E. Enochs and O. M. G. Jenda, Relative Homological Algebra, Walter de Gruyter. Berlin-New York, 2000.

[10] E. E. Enochs, O. M. G. Jenda and J. A. Lopez-Ramos, The existence of Gorenstein flat covers, Math. Scand 94 (1), 46-62, 2004.

[11] S. Jøndrup, p.p.rings and finitely generated flat ideals, Proc. Amer. Math. Soc. 28 (2), 431-435, 1971.

[12] Z. K. Liu, Rings with flat left socles, Comm. Algebra 23 (5), 1645-1656, 1995.

[13] L. X. Mao and N. Q. Ding, FI-injective and FI-flat modules, J. Algebra 309 (1), 367-385, 2007.

[14] L. X. Mao and N. Q. Ding, On divisible and torsionfree modules, Comm. Algebra 36 (2), 708-731, 2008.

[15] L. X. Mao, On mininjective and min-flat modules, Publ. Math. Debrecen 72 (3-4), 347-358, 2008.

[16] W. K. Nicholson and M. F. Yousif, Principally injective rings, J. Algebra 174 (1), 77-93, 1995.

[17] J. J. Rotman, An Introduction to Homological Algebra, Academic press, New Yock, 1979.

[18] E. A. Rutter, Rings with the principle extension property, Comm. Algebra 3 (3), 203-212, 1975.

[19] B. Stenström, Coherent rings and FP-injective modules, J. London Math. Soc. 2, 323-329, 1970.

[20] J. Trlifaj, Cover, envelopes, and cotorsion theories, in:Homological Methods in Module Theory. Lecture notes for the workshop, Cortona, 10-16, 2000.

[21] R. Wisbauer, Foundations of Module and Ring Theory, London-Tokyo: Gordon and Breach 1991.

[22] Y. F. Xiao, Rings with flat socles, Proc. Amer. Math. Soc. 123 (8), 2391-2395, 1995.

[23] J. Z. Xu, Flat Covers of Modules, Lecture Note in Math. Springer-Verlag, BerlinHeidelberg-New York, 1634, 1996.

[24] X. X. Zhang, J. L. Chen and J. Zhang, On $(m, n)$-injective modules and $(m, n)$ coherent rings, Algebra Colloq. 12 (1) , 149-160, 2005.

[25] X. X. Zhang and J. L. Chen, On n-semihereditary and n-coherent rings, Int. Electron. J. Algebra 1, 1-10, 2007.

[26] Z. M. Zhu, On n-coherent rings, $n$-hereditary rings and n-regular rings, Bull. Iranian Math. Soc. 37 (4), 251-267, 2011.

[27] Z. M. Zhu, $\mathscr{C}$-coherent rings, $\mathscr{C}$-semihereditary rings and $\mathscr{C}$-regular rings, Studia Sci. Math. Hungar 50 (4), 491-508, 2013.

[28] Z. M. Zhu, Strongly C C-coherent rings, Math. Rep. 19 (4), 367-380, 2017.

[29] Z. M. Zhu, On П-coherence of rings, Hacet. J. Math. Stat. 46 (5), 875-886, 2017. 